Psychotherapeut 2020 $65: 101-108$ https://doi.org/10.1007/s00278-020-00404-2 Online publiziert: 18. Februar 2020

(c) Der/die Autor(en) 2020
Johanna Schulte · Fanny A. Dietel - Ulrike Buhlmann

Institut für Psychologie, Westfälische Wilhelms-Universität Münster, Münster, Deutschland

\title{
Störungseinsicht bei körperdysmorpher Störung, Zwangsstörung und sozialer Angststörung
}

\author{
Untersuchung eines transdiagnostisch \\ relevanten Merkmals
}

\begin{abstract}
Die körperdysmorphe Störung (KDS) geht oft mit einer geringen bis fehlenden Störungseinsicht einher. Folglich begeben sich viele Betroffene nicht in psychologische oder psychiatrische Behandlungen, sondern beanspruchen nichtpsychologische Behandlungen wie ästhetisch-plastische Verfahren. Im vorliegenden Beitrag wird die Einsicht bei der KDS mit der Einsicht bei der Zwangsstörung (ZS) und sozialen Angststörung (SAS) verglichen.
\end{abstract}

\section{Grundlagen}

Die KDS zeichnet sich durch übermäßige Beschäftigung mit wahrgenommenen Makeln im eigenen Aussehen aus, deren Einschätzung andere Menschen nicht in diesem Ausmaß oder gar nicht teilen (American Psychiatric Association [APA] 2013). Betroffene sorgen sich meistens um einen oder mehrere Aspekte des Gesichts- oder Kopfbereichs (z. B. Größe der Nase, Haut oder Haare); es können jedoch auch andere Körperregionen, wie z.B. der allgemeine Körperbau oder der Intimbereich, im Fokus stehen. Als Bewältigungsversuch werden repetitive Handlungen ausgeführt, z.B. Pflegerituale, Rückversicherungen, Vergleichen des eigenen Aussehens mit dem anderer (z.B. bei Treffen, über soziale Medien oder in Filmen) und das Überprüfen des eigenen Aussehens auf Fotos oder in reflektierenden Oberflächen (z. B. Spiegel, Schaufenster). Die Beschäftigung mit dem Aussehen ist mit deutlichem Leiden oder Beeinträchtigung in sozialen, beruflichen oder anderen wichtigen Lebensbereichen (APA 2013) sowie erhöhter Suizidalität (Angelakis et al. 2016) verbunden.

Die KDS ist mit einer Punktprävalenz von 1,9\% bei Erwachsenen und 2,2\% bei Jugendlichen eine vergleichsweise häufige Erkrankung (Veale et al. 2016). Dennoch werden nur wenige Betroffene entsprechend diagnostiziert und erhalten eine adäquate psychotherapeutische oder psychopharmakologische Behandlung (Buhlmann 2011; Marques et al. 2010). Stattdessen werden häufig medizinische oder kosmetische Behandlungen in Anspruch genommen. Tatsächlich berichten bis zu 76,4\% der Betroffenen, sich zunächst an nichtpsychologische bzw. nichtpsychiatrische Behandler zu wenden (Phillips et al. 2001). Während die KDS-Prävalenz auf 5,8\% in ambulanten und $7,4 \%$ in stationären psychotherapeutischen bzw. psychiatrischen Behandlungsangeboten geschätzt wird, beträgt sie $13,2 \%$ bei Patienten in der plastischen Chirurgie und 11,3\% bei Patienten in Hautarztpraxen (Veale et al. 2016). Kosmetische Maßnahmen zur Veränderung der wahrgenommenen Makel führen jedoch in der Regel nicht zur Verbesserung, sondern oftmals sogar zur Verschlechterung der Symptomatik (Bowyer et al. 2016).

Die Gründe für die niedrigen Erkennungsraten und das mangelnde Hilfesuchverhalten sind vielschichtig. Einerseits stellt die KDS noch immer ein in den Medien unterrepräsentiertes und somit relativ unbekanntes Störungsbild dar. Folglich wissen viele Betroffene nicht, dass sie eine diagnostizier- und behandelbare psychische Störung haben, und berichten nicht von ihren Beschwerden. Hinzu kommt starke Scham bezüglich ihrer Sorgen, die eine Thematisierung zusätzlich erschwert. Darüber hinaus geht die KDS oftmals mit geringer bis fehlender Störungseinsicht einher, d.h., viele Betroffene sind mehr oder weniger davon überzeugt, dass der im Aussehen wahrgenommene Makel tatsächlich physisch vorliegt (Phillips 2004) und ihnen demnach auch nur mit medizinischen oder kosmetischen Behandlungen geholfen werden kann (Buhlmann 2011).

Da die fehlende Störungseinsicht ein derart hervorstechendes Symptom der KDS ist, wurde sie seit der Aufnahme der KDS in die dritte revidierte Version des Diagnostischen und Statistischen Manuals Psychischer Störungen (DSM-III-R; APA 1987) als wahnhafte Störung mit körperbezogenem Wahn berücksichtigt und zusätzlich zur KDS vergeben. Mittlerweile zeigt eine breite Befundlage, dass sich wahnhafte und nicht wahnhafte Betroffene hinsichtlich der meisten 
demografischen und klinischen Merkmale nicht unterscheiden (Phillips et al. 2014). Zudem wird im Rahmen klinischer Beobachtungen angenommen, dass die Einsicht im Störungsverlauf entlang eines Kontinuums variiert (Phillips 2004). Seit dem jüngst eingeführten Spezifizierungskriterium zur Einsicht im DSM-5 (APA 2013) sollte die Diagnose einer wahnhaften Störung bei körperdysmorphen Überzeugungen daher nicht mehr gestellt, sondern der Grad der Einsicht bei Vergabe der KDS als „gut oder angemessen“, „wenig“ oder „fehlend/mit wahnhaften Überzeugungen" bestimmt werden. Berichtet ein Patient demnach beispielsweise, vollständig und starr davon überzeugt zu sein, körperlich entstellt zu sein, weist darüber hinaus jedoch keine psychotischen Merkmale auf, spricht dies für eine Störung mit fehlender Einsicht. Ein ähnliches Vorgehen ist für die ausstehende 11. Version der Internationalen statistischen Klassifikation der Krankheiten und verwandter Gesundheitsprobleme geplant (ICD-11; World Health Organization 2018).

Auch bei Menschen mit ZS können störungsspezifische Überzeugungen einen rigiden Charakter annehmen (APA 2013). Die wenigen bisher vorliegenden Vergleichsstudien bezüglich der Einsicht zwischen KDS und ZS zeigen, dass bei Menschen mit KDS eine vergleichsweise schlechtere Einsicht besteht. Phillips et al. (2012) zeigten, dass von 211 Personen mit ZS $2,4 \%$ als wahnhaft und $28,9 \%$ mit exzellenter Einsicht eingeordnet werden konnten, während bei 68 Personen mit KDS das gegenläufige Muster vorlag, d.h. bei 2,9\% der Betroffenen die Einsicht exzellent und bei $32 \%$ fehlend war. In einer Studie von Toh et al. (2017) wiesen Patienten mit KDS $(n=27)$ und einer psychotischen Störung $(n=20)$ eine signifikant niedrigere Einsicht auf als Patienten mit ZS $(n=19)$ und eine nichtklinische Vergleichsgruppe $(n=42)$. Insgesamt illustrieren die Studien hinsichtlich der Störungseinsicht deutliche Unterschiede zwischen der KDS und ZS, trotz deren gemeinsamer diagnostischer Einordnung unter den Zwangsspektrumstörungen.

Einsicht erscheint auch innerhalb der von SAS Betroffenen besonders inter- essant, v. a., da im DSM-5 die Einsicht in die Übermäßigkeit der Angst kein diagnostisches Kriterium der SAS mehr darstellt (APA 2013). Auch im klinischen Bild überschneidet sich die SAS mit eben den Störungen, die durch unterschiedliche Einsichtsgrade gekennzeichnet sind. So zeigen KDS und SAS Gemeinsamkeiten bezüglich des Störungsverlaufs sowie der Tendenz der Betroffenen, sozialen Situationen und Bewertungsängsten durch Sicherheitsverhaltensweisen zu begegnen oder zu vermeiden (Grocholewski et al. 2013). Die SAS überlappt mit der ZS bezüglich ausgeprägter Angst vor jeweils störungsspezifischen Stimuli; beide Störungen insgesamt sind hochkomorbid (Abramowitz und Deacon 2005). In der einzigen den Autoren des vorliegenden Beitrags bekannten Studie, in der die Einsicht der Teilnehmenden bei vorliegender SAS systematisch untersucht wurde, zeigte sich im Mittel eine nur ausreichende Einsicht in die sozialängstlichen Grundüberzeugungen (Vigne et al. 2014). Trotz der phänomenologischen Nähe dieser Störungen existiert darüber hinaus keine Studie, die dieses Merkmal vergleichend bei KDS, SAS und ZS betrachtet.

Die störungsübergreifende Untersuchung der Einsicht erscheint für die Behandlungsrationale der genannten Störungen relevant. Ihr Grad wird sowohl bei Menschen mit KDS (Greenberg et al. 2019; Phillips et al. 2002) als auch mit ZS (Catapano et al. 2010) und SAS (Vigne et al. 2014) als Prädiktor für ein geringeres Ansprechen auf psychotherapeutische und psychopharmakologische Behandlung diskutiert. Unabhängig von der vorliegenden Störung sind Behandler bei Patienten mit geringer Einsicht vor Behandlungsbeginn und während des Therapieprozesses gefordert, den Überzeugungen adäquat therapeutisch zu begegnen.

\section{Ziel der Arbeit}

Die vorliegende Studie hat zum Ziel, erstmals den Grad der Störungseinsicht und ihrer relevanten Dimensionen, z. B. Überzeugungsstärke und Beziehungsideen, zwischen KDS, ZS und SAS zu vergleichen. Es wird angenommen, dass
Betroffene mit KDS eine signifikant geringere Einsicht aufweisen als Betroffene mit ZS und SAS. Die Ergebnisse bieten wichtige Implikationen für die klinische Einschätzung der störungsspezifischen Überzeugungen und deren Behandlung.

\section{Material und Methode}

\section{Stichprobe}

\section{Rekrutierung der Teilnehmer}

Die Rekrutierung erfolgte über öffentliche Aushänge im Großraum Berlin. Das Projekt erhielt ein positives Votum der lokalen Ethikkommission. Allgemeine Ausschlusskriterien waren eine frühere oder aktuelle psychotische Störung sowie eine frühere oder aktuelle Diagnose der jeweils anderen primären Störungen. Alle Diagnosen psychischer Störungen wurden durch das Strukturierte Klinische Interview für DSM-IV (SKID; Wittchen et al. 1997) erfasst.

\section{Teilnehmende mit körper- dysmorpher Störung}

Die KDS-Gruppe bestand aus 36 Personen mit einer aktuellen KDS-Diagnose. Die Symptomschwere der KDS wurde mithilfe der Modifikation der Yale Brown Obsessive-Compulsive Scale für KDS (BDD-YBOCS; Stangier et al. 2000), einem aus 12 Items bestehenden Fremdbeurteilungsinstrument in Bezug auf die letzte Woche, erfasst. Die interne Konsistenz innerhalb der vorliegenden Gruppe war gut (Cronbachs $a=0,77$ ). Die BDD-YBOCS wies auf eine moderat bis schwer ausgeprägte Symptomatik hin (• Tab. 1). Neben der KDS als primäre Diagnose (basierend auf der aktuellen Symptomschwere) lagen folgende aktuelle Komorbiditäten vor: Major Depression $(n=15)$, spezifische Phobie $(n=12)$, Dysthymie $(n=4)$, Alkoholabhängigkeit $(n=3)$, posttraumatische Belastungsstörung $(n=3)$, Alkoholmissbrauch $(n=2)$, Abhängigkeit von anderen Substanzen $(n=2)$ und hypochondrische Störung, Störung mit Essanfällen, Panikstörung ohne Agoraphobie, Panikstörung mit Agoraphobie, Bipolar-I-Störung (jeweils $n=1)$. 
Psychotherapeut 2020 -65:101-108 https://doi.org/10.1007/s00278-020-00404-2

(c) Der/die Autor(en) 2020

\section{J. Schulte ·F. A. Dietel · U. Buhlmann}

\section{Störungseinsicht bei körperdysmorpher Störung, Zwangsstörung und sozialer Angststörung. Untersuchung eines transdiagnostisch relevanten Merkmals}

\section{Zusammenfassung}

Hintergrund. Die körperdysmorphe Störung (KDS) geht mit einer geringen Störungseinsicht bis zu wahnhaft anmutenden Überzeugungen bezüglich wahrgenommener körperlicher Makel einher, die für andere nur leicht oder nicht sichtbar sind. Während die Einsicht bei der KDS bereits vielfach untersucht wurde und obwohl sie ein transdiagnostisch relevantes Merkmal ist, gibt es kaum vergleichende Studien.

Ziel der Arbeit. Es wird untersucht, inwiefern sich Personen mit einer KDS von Personen mit einer Zwangsstörung (ZS) und einer sozialen Angststörung (SAS) dimensional und kategorial hinsichtlich der Einsicht unterscheiden.
Material und Methode. Personen mit KDS $(n=36)$, ZS $(n=38)$ und SAS $(n=36)$ beantworteten diagnostische Interviews und Fragebogen. Die Einsicht wurde mithilfe eines teilstrukturierten Interviews, das verschiedene Facetten und Grade von Einsicht erfasst, beurteilt (Brown Assessment of Beliefs Scale). Ergebnisse. In allen Gruppen lagen unterschiedliche Einsichtsgrade im gesamten Spektrum von exzellent bis fehlend vor. Bei den Betroffenen mit einer KDS entsprach der Gesamtmittelwert einer schlechten $(M=13,25, S D \pm 6,32)$, bei Betroffenen mit einer SAS einer ausreichenden $(M=10,39$, $S D \pm 5,70)$ und bei Betroffenen mit einer ZS einer guten Einsicht $(M=4,74, S D \pm 5,49)$. Abgesehen von einer stärkeren Fixiertheit der
Überzeugung bei KDS war die Einsicht bei KDS und SAS dimensional und kategorial nicht signifikant voneinander verschieden, jedoch bei beiden Störungen schlechter als bei ZS. Diskussion. Die Ergebnisse bestätigen, dass die KDS mit geringer Einsicht einhergeht, dies jedoch fast gleichermaßen auf die SAS zutrifft. Die Störungseinsicht erscheint bei allen untersuchten Störungen relevant und sollte in der Psychotherapie von Beginn an diagnostisch und psychotherapeutisch berücksichtigt werden.

\section{Schlüsselwörter}

Kognition · Fixiertheit · Überzeugungen · Inanspruchnahme therapeutischer Versorgung . Motivierende Gesprächsführung

\section{Insight in body dysmorphic, obsessive-compulsive and social anxiety disorders. Investigation of a transdiagnostically relevant feature}

\section{Abstract}

Background. Body dysmorphic disorder (BDD) is often characterized by poor insight into the disorder up to delusional beliefs about perceived flaws or defects in one's own physical appearance, which are only slightly or not visible to others. Although insight has often been studied in BDD, research on insight comparing different psychological disorders is sparse.

Objective. This study investigated whether insight in individuals with BDD, obsessivecompulsive disorder (OCD), and social anxiety disorder (SAD) is different with respect to dimensional and categorical perspectives.
Material and methods. Individuals with $\operatorname{BDD}(n=36), \operatorname{OCD}(n=38)$, and SAD $(n=36)$ completed several diagnostic interviews and questionnaires. Insight was assessed using a semi-structured interview measuring different facets and degrees of insight (Brown Assessment of Beliefs Scale).

Results. All clinical groups reported different degrees of insight in the complete spectrum from excellent to absent. Mean sum scores corresponded to poor insight in persons with $\operatorname{BDD}(M=13.25, S D \pm 6.32)$, fair insight in $S A D$ $(M=10.39, S D \pm 5.70)$ and good insight in $O C D$ $(M=4.74, S D \pm 5.49)$. Apart from a stronger fixity of beliefs in BDD, insight in BDD and
SAD was dimensionally and categorically not significantly different but in both disorders poorer than in OCD.

Discussion. The findings confirmed that BDD is associated with poor insight; however, this applied almost equally to SAD. Insight into the disorder seems relevant in every disorder investigated in this study and should be taken into consideration in the diagnostics and psychotherapy from the very beginning.

\section{Keywords}

Cognition · Fixity · Beliefs $\cdot$ Health care utilization $\cdot$ Motivational interviewing

\section{Teilnehmende mit Zwangsstörung} Die ZS-Gruppe bestand aus 38 Personen mit der aktuellen Diagnose einer ZS. Der Symptomschweregrad der ZS wurde mithilfe der Yale Brown Obsessive-Compulsive Scale (YBOCS; Hand und BüttnerWestphal 1991) erfasst. Die YBOCS ist ein halbstrukturiertes Fremdbeurteilungsinstrument, das die Symptomschwere der ZS innerhalb der letzten Woche mithilfe von 10 Items erfasst. Die interne Konsistenz innerhalb der vorliegenden Gruppe war sehr gut $(\alpha=0,89)$. Die YBOCS-In- terviews ergaben eine moderate bis extreme Symptomschwere (- Tab. 1). Neben der primären ZS lagen als aktuelle Komorbiditäten vor: Major Depression $(n=9)$, spezifische Phobie $(n=5), \mathrm{Pa}-$ nikstörung ohne Agoraphobie $(n=2)$ sowie Dysthymie, Alkoholmissbrauch, hypochondrische Störung, nicht näher bezeichnete Essstörung und Tourette-Syndrom (jeweils $n=1$ ).

\section{Teilnehmende mit sozialer Angststörung}

Die SAS-Gruppe bestand aus 36 Personen mit einer aktuellen SAS-Diagnose. Die sozialängstlichen Symptome wurden mithilfe der Liebowitz Social Anxiety Scale (LSAS; Stangier und Heidenreich 2004) in Bezug auf die letzte Woche erfasst. Die LSAS besteht aus 24 Items, die jeweils eine soziale Situation beschreiben, die vom Probanden im Selbstbericht bezüglich Angst und Vermeidung bewertet wird und deren Angaben zu einem 
Tab. 1 Demografische und klinische Merkmale der Stichproben

\begin{tabular}{|c|c|c|c|c|c|}
\hline & $\begin{array}{l}\text { KDS } \\
(n=36)\end{array}$ & $\begin{array}{l}\text { ZS } \\
(n=38)\end{array}$ & $\begin{array}{l}\text { SAS } \\
(n=36)\end{array}$ & Statistik $^{a}$ & $p$ \\
\hline Alter (Jahre, $M \pm S D$ ) & $\begin{array}{l}33,06 \\
( \pm 11,09)\end{array}$ & $\begin{array}{l}37,11 \\
( \pm 10,93)\end{array}$ & $\begin{array}{l}33,61 \\
( \pm 9,71)\end{array}$ & $F(2,107)=1,60$ & 0,207 \\
\hline $\begin{array}{l}\text { Weibliches Geschlecht } \\
(\%, n)\end{array}$ & $63,9(23)$ & $44,7(17)$ & $61,1(22)$ & $X^{2}(2,110)=3,25$ & 0,197 \\
\hline Alleinstehend $(\%, n)$ & $36,1(13)$ & $57,9(22)$ & $47,2(17)$ & $X^{2}(2,110)=3,52$ & 0,172 \\
\hline $\begin{array}{l}\text { Höchster Abschluss } \\
(\%, n)\end{array}$ & - & - & - & - & 0,834 \\
\hline Hauptschule & $2,8(1)$ & $2,6(1)$ & $5,6(2)$ & - & - \\
\hline Realschule & $16,7(6)$ & $31,6(12)$ & $13,9(5)$ & - & - \\
\hline Fachabitur & $16,7(6)$ & $10,5(4)$ & $13,9(5)$ & - & - \\
\hline Abitur & $33,3(12)$ & $26,3(10)$ & $27,8(10)$ & - & - \\
\hline Fachhochschule & $13,9(5)$ & $7,9(3)$ & $16,7(6)$ & - & - \\
\hline Universität & $16,7(6)$ & $18,4(7)$ & $16,7(6)$ & - & - \\
\hline Anderer & $0,0(0)$ & $2,63(1)$ & $5,6(2)$ & - & - \\
\hline $\begin{array}{l}\text { Aktuelle Psychotherapie } \\
(\%, n)\end{array}$ & $27,8(10)$ & $73,7(28)$ & $33,3(12)$ & $X^{2}(2,110)=18,88$ & $<0,001$ \\
\hline $\begin{array}{l}\text { Frühere Psychotherapie } \\
(\%, n)\end{array}$ & $55,6(20)$ & $81,6(31)$ & $77,1(27)^{b}$ & $X^{2}(2,110)=6,94$ & 0,031 \\
\hline $\begin{array}{l}\text { Ersterkrankungsalter } \\
\text { (Jahre; } M \pm S D \text { ) }\end{array}$ & $15,5( \pm 8,23)$ & $\begin{array}{l}18,5 \\
( \pm 10,54)\end{array}$ & $\begin{array}{l}14,69 \\
( \pm 6,82)\end{array}$ & $F(2,107)=1,96$ & 0,146 \\
\hline$B D I-I I(M \pm S D)$ & $\begin{array}{l}20,25 \\
( \pm 11,23)\end{array}$ & $\begin{array}{l}16,55 \\
( \pm 11,31)\end{array}$ & $\begin{array}{l}15,42 \\
( \pm 8,11)\end{array}$ & $F(2,107)=2,16$ & 0,121 \\
\hline$B D D-Y B O C S(M \pm S D)$ & $\begin{array}{l}29,03 \\
( \pm 6,13)\end{array}$ & - & - & - & - \\
\hline$Y B O C S(M \pm S D)$ & - & $\begin{array}{l}30,74 \\
( \pm 8,06)\end{array}$ & - & - & - \\
\hline $\operatorname{LSAS}(M \pm S D)$ & - & - & $\begin{array}{l}76,93 \\
( \pm 23,38)\end{array}$ & - & - \\
\hline \multicolumn{6}{|c|}{$\begin{array}{l}\text { BDD-YBOCS Modifikation der Yale Brown Obsessive-Compulsive Scale für KDS (Stangier et al. 2000), } \\
\text { BDI-II Beck Depressions-Inventar-II, revidierte Version (Hautzinger et al. 2006), KDS körperdysmor- } \\
\text { phe Störung, LSAS Liebowitz Social Anxiety Scale (Stangier und Heidenreich 2004), SAS soziale } \\
\text { Angststörung, YBOCS Yale Brown Obsessive-Compulsive Scale (Hand und Büttner-Westphal 1991), } \\
\text { ZS Zwangsstörung } \\
\text { "Analysis of variance" (ANOVA), X'-Test oder Exakter Test nach Fisher } \\
\text { "Die Angabe lag bei } n=35 \text { der SAS-Gruppe vor }\end{array}$} \\
\hline
\end{tabular}

Gesamtwert addiert werden. Die interne Konsistenz war in der vorliegenden SAS Gruppe exzellent $(\alpha=0,94)$. Die LSAS wies auf eine mittel bis schwer ausgeprägte Symptomatik hin (• Tab. 1). Neben der primären SAS lagen aktuelle Komorbiditäten vor: spezifische Phobie $(n=11)$, Alkoholmissbrauch $(n=5)$, Major Depression $(n=4)$, Dysthymie $(n=4)$, Alkoholabhängigkeit $(n=4)$, Abhängigkeit von anderen Substanzen $(n=2)$, Panikstörung ohne Agoraphobie $(n=2), \mathrm{Pa}$ nikstörung mit Agoraphobie und posttraumatische Belastungsstörung (jeweils $n=1)$.

\section{Erhebungsinstrumente}

\section{Depressivität}

Das Beck Depressionsinventar-II (BDIII) erfasst die Schwere der depressiven Symptomatik innerhalb der letzten 2 Wochen (Hautzinger et al. 2006). Höhere Werte weisen auf eine höhere Symptomschwere hin (Spanne des Gesamtwerts 0-63). Die interne Konsistenz war hoch $(\alpha=0,89)$.

\section{Einsicht}

Die Störungseinsicht wurde mithilfe der Brown Assessment of Beliefs Scale (BABS; Buhlmann 2014) erfasst. Dieses teilstrukturierte Interview wurde ent- wickelt, um die Einsicht in die jeweils zugrunde liegende Überzeugung oder Annahme bei verschiedenen Störungsbildern $\mathrm{zu}$ quantifizieren (Eisen et al. 1998). Dafür wird eine möglichst spezifische Grundüberzeugung ausgewählt, z. B. „Ich sehe hässlich aus“ bei vorliegender KDS, „Ich wirke peinlich“ bei vorliegender SAS oder „Ich muss den Herd kontrollieren, bis ich vollkommen sicher bin, sonst brennt die Wohnung ab“ bei vorliegender ZS. Für die letzte Woche werden folgende Dimensionen in Bezug auf die Grundüberzeugung beurteilt: 1) Überzeugung bzw. Sicherheit hinsichtlich der Richtigkeit, 2) Einschätzung der Wahrnehmung der Meinung anderer Personen, 3) Erklärung ggf. abweichender Auffassungen, 4) Fixiertheit der eigenen Überzeugung bzw. Zögern bei Hinterfragen, 5) Versuche, die eigene Überzeugung $z u$ widerlegen, 6) Anerkennen einer psychologischen oder psychiatrischen Ursache der Überzeugung (benannt als Einsicht im engeren Sinne). Ein siebtes Item erfasst Beziehungsideen, geht jedoch nicht in die Gesamtbeurteilung oder den Summenwert ein. Die Dimensionen werden jeweils anhand einer Likert-Skala von 0 bis 4 mit unterschiedlichen Ankern pro Item eingeschätzt, z. B. Item 1 Wert 0 „vollkommen sicher, dass Überzeugungen falsch sind (0\% Sicherheit)“. Der Summenwert der Items wird als globales Maß der Einsicht gewertet; höhere Werte zeigen geringere Einsicht an. Die BABS ermöglicht eine Einteilung des Einsichtsgrades anhand des Gesamtwerts in 4 Kategorien (Phillips et al. 2012): Gesamtwert 0-3: exzellente Einsicht; Gesamtwert 4-7: gute Einsicht; Gesamtwert 8-12: ausreichende Einsicht; Gesamtwert 13-17 oder $\geq 18$ und Überzeugung (Item 1) Wert $\leq 3$ : schlechte Einsicht; Gesamtwert $\geq 18$ und Überzeugung (Item 1) Wert = 4: fehlende Einsicht/wahnhaft. Die interne Konsistenz in der vorliegenden Stichprobe war exzellent $(\alpha=0,90)$.

\section{Studienablauf}

Die vorliegende Studie war Teil eines umfangreicheren, durch die Deutsche Forschungsgemeinschaft (DFG) geförderten Forschungsvorhabens zur KDS, das an 


\begin{tabular}{|c|c|c|c|c|c|c|c|}
\hline & \multicolumn{3}{|l|}{$M(S D) / \%(n)$} & \multirow[t]{2}{*}{ Statistik $^{\mathbf{a}}$} & \multirow[t]{2}{*}{$p$} & \multirow{2}{*}{$\begin{array}{l}\text { Effekt- } \\
\text { stärke }^{b}\end{array}$} & \multirow[t]{2}{*}{ Post-hoc-Test $^{c}$} \\
\hline & $\begin{array}{l}\text { KDS } \\
(n=36)\end{array}$ & $\begin{array}{l}\text { ZS } \\
(n=38)\end{array}$ & $\begin{array}{l}\text { SAS } \\
(n=36)\end{array}$ & & & & \\
\hline$B A B S$, gesamt $(M \pm S D)^{d}$ & $\begin{array}{l}13,25 \\
( \pm 6,32)\end{array}$ & $\begin{array}{l}4,74 \\
( \pm 5,49)\end{array}$ & $\begin{array}{l}10,39 \\
( \pm 5,70)\end{array}$ & $F(2,107)=20,44$ & $<0,001$ & 0,28 & $\begin{array}{l}\mathrm{KDS}>\mathrm{ZS}, \mathrm{KDS}=\mathrm{SAS}, \\
\mathrm{SAS}>\mathrm{ZS}\end{array}$ \\
\hline Überzeugung $(\mathrm{M} \pm \mathrm{SD})$ & $2,67( \pm 1,12)$ & $\begin{array}{l}1,29 \\
( \pm 1,27)\end{array}$ & $2,31( \pm 1,14)$ & $F(2,107)=13,59$ & $<0,001$ & 0,20 & $\begin{array}{l}\mathrm{KDS}>\mathrm{ZS}, \mathrm{KDS}=\mathrm{SAS}, \\
\mathrm{SAS}>\mathrm{ZS}\end{array}$ \\
\hline $\begin{array}{l}\text { Wahrnehmung der Meinung } \\
\text { anderer }(\mathrm{M} \pm \mathrm{SD})\end{array}$ & $1,72( \pm 1,23)$ & $\begin{array}{l}0,39 \\
( \pm 0,86)\end{array}$ & $1,64( \pm 1,18)$ & $F(2,68,24)=20,78$ & $<0,001$ & 0,24 & $\begin{array}{l}\mathrm{KDS}>\mathrm{ZS}, \mathrm{KDS}=\mathrm{SAS}, \\
\mathrm{SAS}>\mathrm{ZS}\end{array}$ \\
\hline $\begin{array}{l}\text { Erklärung von abweichenden } \\
\text { Auffassungen }(\mathrm{M} \pm \mathrm{SD})\end{array}$ & $2,33( \pm 1,20)$ & $\begin{array}{l}0,82 \\
( \pm 1,14)\end{array}$ & $2,08( \pm 1,27)$ & $F(2,107)=17,10$ & $<0,001$ & 0,24 & $\begin{array}{l}\mathrm{KDS}>\mathrm{ZS}, \mathrm{KDS}=\mathrm{SAS}, \\
\mathrm{SAS}>\mathrm{ZS}\end{array}$ \\
\hline Fixiertheit $(M \pm S D)$ & $2,64( \pm 1,42)$ & $\begin{array}{l}0,87 \\
( \pm 1,26)\end{array}$ & $1,58( \pm 1,32)$ & $F(2,107)=16,52$ & $<0,001$ & 0,24 & $\begin{array}{l}\mathrm{KDS}>\mathrm{ZS}, \mathrm{KDS}>\mathrm{SAS}, \\
\mathrm{SAS}=\mathrm{ZS}\end{array}$ \\
\hline $\begin{array}{l}\text { Versuch, Überzeugung zu } \\
\text { widerlegen }(M \pm S D)\end{array}$ & $2,36( \pm 1,43)$ & $\begin{array}{l}0,92 \\
( \pm 1,38)\end{array}$ & $1,89( \pm 1,39)$ & $F(2,107)=10,18$ & $<0,001$ & 0,16 & $\begin{array}{l}\mathrm{KDS}>\mathrm{ZS}, \mathrm{KDS}=\mathrm{SAS}, \\
\mathrm{SAS}>\mathrm{ZS}\end{array}$ \\
\hline $\begin{array}{l}\text { Einsicht in psychische Ursa- } \\
\text { che }(M \pm S D)\end{array}$ & $1,53( \pm 1,46)$ & $\begin{array}{l}0,45 \\
( \pm 0,98)\end{array}$ & $0,89( \pm 1,21)$ & $F(2,68,56)=6,95$ & 0,001 & 0,12 & $\begin{array}{l}\mathrm{KDS}>\mathrm{ZS}, \mathrm{KDS}=\mathrm{SAS}, \\
\mathrm{SAS}=\mathrm{ZS}\end{array}$ \\
\hline Beziehungsideen $(\mathrm{M} \pm \mathrm{SD})$ & $1,72( \pm 1,49)$ & $\begin{array}{l}0,47 \\
( \pm 0,98)\end{array}$ & $1,44( \pm 1,54)$ & $F(2,66,77)=11,10$ & $<0,001$ & 0,14 & $\begin{array}{l}\mathrm{KDS}>\mathrm{ZS}, \mathrm{KDS}=\mathrm{SAS}, \\
\mathrm{SAS}>\mathrm{ZS}\end{array}$ \\
\hline Grad der Einsicht $^{e}$ & - & - & - & - & $<0,001$ & 0,39 & - \\
\hline Exzellent $(\%, n)$ & $11,1(4)$ & $52,6(20)$ & $16,7(6)$ & - & - & - & - \\
\hline Gut $(\%, n)$ & $5,6(2)$ & $15,8(6)$ & $16,7(6)$ & - & - & - & - \\
\hline Ausreichend $(\%, n)$ & $25,0(9)$ & $26,3(10)$ & $22,2(8)$ & - & - & - & - \\
\hline Schlecht $(\%, n)$ & $44,4(16)$ & $2,6(1)$ & $38,9(14)$ & - & - & - & - \\
\hline Fehlend/wahnhaft $(\%, n)$ & $13,9(5)$ & $2,6(1)$ & $5,6(2)$ & - & - & - & - \\
\hline \multicolumn{8}{|c|}{$\begin{array}{l}\text { BABS Brown Assessment of Beliefs Scale (Buhlmann 2014), KDS körperdysmorphe Störung, SAS Soziale Angststörung, ZS Zwangsstörung } \\
\text { "Analysis of variance" (ANOVA; BABS) oder Exakter Test nach Fisher (Grad der Einsicht) } \\
\text { "Partielles n oder Cramers V } \\
\text { "Bonferroni-korrigiert } \\
\text { "Item „Beziehungsideen" nicht im Summenwert enthalten } \\
\text { "Einteilung nach Gesamtwerten: 0-3: exzellente Einsicht; 4-7: gute Einsicht; 8-12: ausreichende Einsicht; } 13-17 \text { oder } \geq 18 \text { und Wert } \leq 3 \text { bei Item } 1 \\
\text { "Überzeugung": schlechte Einsicht; } \geq 18 \text { und Wert } 4 \text { bei Item } 1 \text { „Überzeugung": fehlende Einsicht/wahnhaft }\end{array}$} \\
\hline
\end{tabular}

3 Untersuchungstagen stattfand (Buhlmann et al. 2015). Am ersten Tag wurden, nach umfangreicher Studieninformation und Einholung der schriftlichen Einverständniserklärung, die Interviews durchgeführt und Fragebogen ausgefüllt. Nach Beendigung der Studie wurden die Teilnehmer über die Studienziele und -hypothesen aufgeklärt und erhielten eine finanzielle Entschädigung von $8 € / \mathrm{h}$.

\section{Statistische Auswertung}

Die statistische Auswertung erfolgte mithilfe von IBM SPSS Statistics (Version 24). Gruppenvergleiche in Bezug auf Alter, Störungsbeginn, Depressivität sowie einzelne Items und Summenwert der Einsicht wurden mithilfe einfaktorieller Varianzanalysen mit Bonferroni-korrigierten Post-hoc-Tests durchgeführt. Bei nichtvorliegender Varianzhomoge- nität wurde die Teststatistik nach Welch korrigiert. Die Gruppenunterschiede in kategorialen Variablen wurden mithilfe des Pearson $\chi^{2}$-Tests oder, im Fall von $n<5$ in einzelnen Kategorien, dem Exakten Test nach Fisher berechnet. Es wurden jeweils entsprechende Effektstärken berechnet (Cramers V, partielles $\left.\eta^{2}\right)$.

\section{Ergebnisse}

\section{Demografische und klinische Merkmale}

Die Gruppen unterschieden sich nicht hinsichtlich demografischer Merkmale, der Depressivität oder des Alters bei Störungsbeginn (• Tab. 1). Personen mit KDS und SAS befanden sich zum Durchführungszeitpunkt signifikant weniger häufig in ambulanter Psychotherapie.
Dies traf für KDS auch für den Anteil der Personen mit früheren Therapien zu.

\section{Einsicht im Störungsvergleich}

Im mittleren Gesamtwert äußerten die Personen mit KDS eine schlechte, die Personen mit SAS eine ausreichende und die Personen mit ZS eine gute Störungseinsicht (• Tab. 2). Obwohl der Gesamtwert in der KDS-Gruppe numerisch höher war, war er nicht signifikant von dem Gesamtwert in der SAS Gruppe verschieden. Die mittlere Einsicht zeigte sich jedoch sowohl bei vorliegender KDS als auch vorliegender SAS signifikant höher als bei ZS.

Hinsichtlich der einzelnen Dimensionen ergaben sich im Gruppenvergleich durchweg höhere Werte bei Teilnehmenden mit KDS als bei denen mit ZS. Im Vergleich von KDS und SAS lagen hingegen 
gleich hoch ausgeprägte Werte hinsichtlich aller Dimensionen außer bei Fixiertheit vor. Auch im Vergleich von SAS und ZS wies die SA-Gruppe durchweg höhere Werte als die ZS-Gruppe in fast allen Dimensionen auf; nur hinsichtlich der Fixiertheit und Einsicht in eine psychologische/psychiatrische Ursache lagen gleichermaßen niedrige Werte vor. Die Effektstärken der Gruppenvergleiche waren für alle Dimensionen als groß einzuschätzen, mit Ausnahme einer mittleren Effektstärke hinsichtlich der Einsicht in eine psychologische/psychiatrische Ursache.

Auch bezüglich der kategorialen Einschätzung des Einsichtsgrades lagen zwischen den Gruppen signifikant unterschiedliche Verteilungen mit großer Effektstärke vor: Die Einsicht der meisten Personen mit KDS und SAS konnte als schlecht oder fehlend, in der ZS-Gruppe jedoch als exzellent eingeordnet werden. In jeder Gruppe waren alle Kategorien belegt.

\section{Diskussion}

\section{Interpretation der Ergebnisse}

Die vorliegende Studie untersuchte die störungsspezifische Einsicht bei vorliegender KDS im Vergleich zu zwei klinischen Gruppen, spezifisch SAS und ZS. Es ergab sich ein differenziertes Gesamtbild. Die KDS- und SAS-Gruppen zeigten erhöhte Gesamt- und Einzelwerte in der BABS - d.h. sowohl global als auch hinsichtlich der einzelnen Facetten - weniger Einsicht im Vergleich zu der ZS-Gruppe. Personen mit KDS und SAS unterschieden sich bezüglich dieser Werte großteilig nicht signifikant. Eine Ausnahme bildete die Fixiertheit, in der die KDS-Gruppe höhere Werte als die SAS- und ZS-Gruppen aufwies. Bei Teilnehmenden mit SAS und ZS hingegen war diese, ebenso wie die Annahme einer psychologischen Ursache, gleich ausgeprägt, d.h., SAS- und ZS-Betroffene waren gleichsam empfänglich dafür, die eigene Überzeugung anzuzweifeln und eine psychologische Ursache anzuerkennen. In der KDS- und der SAS-Gruppe ergaben sich in der kategorialen Auswertung ähnliche Ergebnismuster im Sinne einer mehrheitlich schlechten bis fehlenden Einsicht. Hingegen war die Einsicht der Teilnehmenden bei vorliegender ZS in mehr als der Hälfte der Fälle exzellent, und jeweils nur vereinzelt als schlecht oder fehlend einzuschätzen.

Diese Ergebnisse lassen sich in den bestehenden empirischen und theoretischen Zusammenhang einordnen. Der Befund einer dimensional und kategorial schlechteren Einsicht bei Betroffenen mit einer KDS, in Kontrast zu einem gegenläufigen Muster bei Betroffenen mit einer ZS, bestätigt die Ergebnisse bisheriger Studien (Phillips et al. 2012; Toh et al. 2017). Im Vergleich zu ZS- scheint die Einsicht bei KDS-Betroffenen demnach deutlich häufiger schlecht ausgeprägt zu sein. Sowohl Personen mit KDS als auch Personen mit SAS erscheinen hingegen gleichermaßen stark von ihren eigenen Annahmen und deren Wahrheitsgehalt überzeugt. Es wurde zuvor erwartet, dass die Einsicht bei KDS-Betroffenen deutlich schlechter als bei der SAS-Betroffenen sein würde, was sich nicht bestätigte. Das Ergebnis könnte durch die gemeinsam zugrunde liegende Bewertungsangst bedingt sein, die sich bei vorliegender KDS auf das Aussehen und bei vorliegender SAS auf das Verhalten oder Angstsymptome bezieht (APA 2013). Da diese Grundüberzeugungen vom Urteil anderer Personen abhängen, können sie bei KDS und SAS nur schwer hinsichtlich ihrer Gültigkeit geprüft werden. Bei Betroffenen mit einer ZS hingegen sind die befürchteten Konsequenzen leichter durch beispielsweise Wissen oder Beobachtung widerlegbar (z. B. ausgeschalteter Herd durch einmaliges Betätigen eines Schalters). Die geringere Einsicht in eine psychologische Ursache, die bei KDS vorlag, könnte für Personen mit KDS durch den eindeutigen körperlichen Bezug und die mit der KDS einhergehenden verzerrten Wahrnehmung erschwert sein (im Sinne von "Ich sehe es, also ist es so“; Kollei und Martin 2010).

Insgesamt ist auffällig, dass die Gesamtwerte der BABS in diesen Stichproben niedriger ausfallen als in vergleichbaren Untersuchungen, insbesondere für KDS- und ZS-Betroffene, für die in anderen Studien die Gesamtwerte über 16 bei vorliegender KDS bezie- hungsweise über 7 bei vorliegender ZS betrugen (Eisen et al. 1998; Phillips et al. 2012; Toh et al. 2017). Gleichzeitig weicht der durchschnittliche Schweregrad der Störung in den hier untersuchten klinischen Gruppen nicht von denen in anderen Studien ab. Die Unterschiede lassen sich damit potenziell durch die Stichprobengewinnung und die damit gegebenen Merkmale (z. B. freie Rekrutierung vs. Behandlungseinrichtung) sowie ggf. die Durchführung des BABS-Interviews erklären (z. B. Auswahl der Grundüberzeugung).

\section{Limitationen der Studie}

Insbesondere die Stichprobengröße ist potenziell zu gering, um tatsächliche kleine Effekte in Unterschieden zwischen KDS und SAS festzustellen. Somit besteht die Möglichkeit, dass sich diese beiden Störungen bezüglich ihrer Einsichtsmuster noch differenzierter unterscheiden. Zukünftige Untersuchungen sollten die Befunde daher in größeren Stichproben replizieren. Eine weitere Limitation besteht darin, dass sich in der vorliegenden Stichprobe zum Erhebungszeitpunkt signifikant mehr Personen in der ZS- vs. SAund KDS-Gruppe in psychotherapeutischer Behandlung befanden. Insbesondere in psychotherapeutischen Behandlungen werden Patienten, z. B. im Rahmen kognitiver Umstrukturierung, dazu angeleitet, eigene Befürchtungen zu hinterfragen und durch realistischere Annahmen zu ersetzen. Die festgestellte bessere Einsicht in der ZS-Gruppe könnte hierdurch verzerrt sein. Obgleich ein Spektrum an Personen mit unterschiedlichem Behandlungsstatus die Repräsentativität erhöht, sollten zukünftige Studien diesen Faktor über Vergleiche in größeren Stichproben beleuchten.

\section{Implikationen für den psychotherapeutischen Umgang mit der Störungseinsicht}

In allen 3 Störungsgruppen gab es Personen, deren Überzeugung durch fehlende Einsicht gekennzeichnet war. Behandler sollten dies entsprechend als Variante der zugrunde liegenden Störungen diagnostisch einordnen; auf die Vergabe 
einer wahnhaften Störung wird ohne Vorliegen anderer psychotischer Symptome verzichtet. Die Einsicht sollte im Sinne eines transdiagnostisch relevanten Faktors in die Behandlung der Störungen einbezogen werden, um die Therapieeffektivität $\mathrm{zu}$ fördern. Alle einzelnen Facetten der Einsicht stellen Ansätze für das therapeutische Arbeiten dar. Bislang sind uns keine Untersuchungen darüber bekannt, welche einzelnen Facetten besonders entscheidend für den Behandlungserfolg sind. In der Praxis sollten daher alle Facetten, z.B. mithilfe der BABS, erhoben und je nach individueller Relevanz bearbeitet werden. Eine besondere Bedeutung kommt sicherlich der Einsicht in eine psychologische oder psychiatrische Ursache (Item 6) zu. Fehlt diese, erscheinen bereits das Aufsuchen eines Psychotherapeuten oder Psychiaters nicht naheliegend sowie Ambivalenz und Widerstand in der Behandlung nachvollziehbar. Es muss dann auf eine Verschiebung der Problemdefinition beim Patienten hingearbeitet werden. Hinsichtlich der KDS bedeutet dies, das wahrgenommene „Aussehensproblem“ als „Wahrnehmungs- oder Körperbildproblem“ zu definieren. Betroffene sollten ab dem Erstgespräch mithilfe motivierender Gesprächsführung darin gefördert werden, sich für eine Psychotherapie und ggf. gegen kosmetische Behandlungen entscheiden $\mathrm{zu}$ können. Zudem sollte in der kognitiven Umstrukturierung bei Patienten mit KDS selbst bei einem leicht sichtbaren Makel nicht über das Vorhandensein oder Ausmaß des Makels diskutiert, sondern dessen Wichtigkeit hinterfragt werden. Dieses Vorgehen erscheint analog zu der üblichen Disputation der Bedeutung von sichtbaren körperlichen Angstsymptomen bei SAS-Patienten oder der Toleranz von Unsicherheit bei ZS-Patienten. Da eine geringe Einsicht in die störungsspezifischen Grundüberzeugungen bei der KDS besonders relevant erscheint, aber auch bei anderen psychischen Störungen wie ZS und SAS auftritt, sollten sich Behandler ermutigt fühlen, bekannte transdiagnostische Strategien in der Psychotherapie anzuwenden.

\section{Fazit für die Praxis}

- Störungseinsicht ist ein transdiagnostisch relevantes Merkmal von Psychopathologie.

- Geringe Störungseinsicht zeichnet sich durch starke Überzeugungen aus; mithilfe der Brown Assessment of Beliefs Scale können unterschiedliche Facetten erfasst werden.

- Sowohl Personen mit einer körperdysmorphen Störung (KDS), einer sozialen Angststörung (SAS) als auch einer Zwangsstörung (ZS) können unterschiedliche Grade der Einsicht aufweisen (exzellent, gut, ausreichend, schlecht, fehlend).

- Bei Betroffenen mit einer KDS oder SAS scheint meist eine schlechte Einsicht vorzuliegen.

- Wenn notwendig, sollte bei Betroffenen mit KDS die Problemdefinition von einem Problem wegen des Aussehens zu einem Problem der Wahrnehmung oder des Körperbilds verschoben werden.

- Da Personen mit einer KDS gegenüber einer Psychotherapie ambivalent eingestellt sind, sollte vom Erstgespräch an die motivierende Gesprächsführung genutzt werden.

- Ambivalenz und Widerstand können Zeichen geringer Einsicht sein; ihnen sollte mit entsprechenden transdiagnostischen Interventionen begegnet werden.

\section{Korrespondenzadresse}

\section{Ulrike Buhlmann}

Institut für Psychologie, Westfälische WilhelmsUniversität Münster

Fliednerstraße 21, 48149 Münster, Deutschland ulrike.buhlmann@wwu.de

Funding. Open Access funding provided by Projekt DEAL.

\section{Einhaltung ethischer Richtlinien}

Interessenkonflikt. J. Schulte, F.A. Dietel und U. Buhlmann geben an, dass kein Interessenkonflikt besteht.

Für diesen Beitrag wurden von den Autoren keine Studien an Tieren durchgeführt. Für die aufgeführten Studien gelten die jeweils dort angegebenen ethischen Richtlinien. Von allen an der Befragung Beteiligten liegt eine schriftliche Einverständniserklärung vor. Die Zustimmung der zuständigen Ethikkommission liegt vor.

Open Access. Dieser Artikel wird unter der Creative Commons Namensnennung 4.0 International Lizenz veröffentlicht, welche die Nutzung, Vervielfältigung, Bearbeitung, Verbreitung und Wiedergabe in jeglichem Medium und Format erlaubt, sofern Sie den/die ursprünglichen Autor(en) und die Quelle ordnungsgemäß nennen, einen Link zur Creative Commons Lizenz beifügen und angeben, ob Änderungen vorgenommen wurden.

Die in diesem Artikel enthaltenen Bilder und sonstiges Drittmaterial unterliegen ebenfalls der genannten Creative Commons Lizenz, sofern sich aus der Abbildungslegende nichts anderes ergibt. Sofern das betreffende Material nicht unter der genannten Creative Commons Lizenz steht und die betreffende Handlung nicht nach gesetzlichen Vorschriften erlaubt ist, ist für die oben aufgeführten Weiterverwendungen des Materials die Einwilligung des jeweiligen Rechteinhabers einzuholen.

Weitere Details zur Lizenz entnehmen Sie bitte der Lizenzinformation auf http://creativecommons.org/ licenses/by/4.0/deed.de.

\section{Literatur}

Abramowitz JS, Deacon BJ (2005) Obsessive-compulsive disorder: Essential phenomenology and overlap with other anxiety disorders. In: Abramowitz JS, Houts AC (Hrsg) Concepts and controversies in obsessive-compulsive disorder. Springer, Heidelberg, Berlin, New York, S 119-149

American Psychiatric Association (1987) Diagnostic and statistical manual of mental disorders, 3.Aufl. American Psychiatric Association, Washington

American Psychiatric Association (2013) Diagnostic and statistical manual of mental disorders, 5. Aufl. American Psychiatric, Washington

Angelakis I, Gooding PA, Panagioti M (2016) Suicidality in body dysmorphic disorder (BDD): a systematic review with meta-analysis. Clin Psychol Rev 49:55-66

Bowyer L, Krebs G, Mataix-Cols D et al (2016) A critical review of cosmetic treatment outcomes in body dysmorphic disorder. Body Image 19:1-8

Buhlmann U (2011) Treatment barriers for individuals with body dysmorphic disorder: an internet survey. J Nerv Ment Dis 199:268-271

Buhlmann U (2014) The German version of the Brown Assessment ofBeliefs Scale(BABS): development and evaluation of its psychometric properties. Compr Psychiatry 55:1968-1971

Buhlmann U, Wacker R, Dziobek I (2015) Inferring other people's states of mind: comparison across social anxiety, body dysmorphic, and obsessive-compulsive disorders. J Anxiety Disord 34:107-113

Catapano F, Perris F, Fabrazzo M et al (2010) Obsessive-compulsive disorder with poor insight: a three-year prospective study. Prog Neuropsychopharmacol Biol Psychiatry 34:323-330

Eisen JL, Phillips KA, Baer L et al (1998) The Brown assessment of beliefs scale: reliability and validity. Am J Psychiatry 155:102-108

Greenberg JL, Phillips KA, Steketee G et al (2019) Predictors of response to cognitive-behavioral 
therapy for body dysmorphic disorder. Behav Ther 50:839-849

Grocholewski A, Kliem S, Heinrichs N (2013) Möglichkeiten zur klinischen Differenzierung von körperdysmorpher Störung und sozialer Angststörung. Z Klin Psychol Psychother 42:163-171

Hand I, Büttner-Westphal H (1991) Die Yale-Brown Obsessive Compulsive Scale (Y-BOCS): Ein halbstrukturiertes Interview zur Beurteilung des Schweregrades von Denk- und Handlungszwängen. Verhaltenstherapie 1:223-225

Hautzinger M, Keller F, Kühner C (2006) Beck Depressions-Inventar: BDI II. Revision. Harcourt Test Services, Frankfurt a.M.

Kollei I, Martin A (2010) Körperdysmorphe Störung: Symptome, Diagnose und Therapie. Psychotherapeut 55:153-166

Marques L, LeBlanc NJ, Weingarden HM et al (2010) Barriers to treatment and service utilization in an internet sample of individuals with obsessive-compulsive symptoms. Depress Anxiety 27:470-475

Phillips KA (2004) Psychosis in body dysmorphic disorder.J Psychiatr Res 38:63-72

Phillips KA, Pinto A, Hart AS et al (2012) A comparison of insight in body dysmorphic disorder and obsessive-compulsive disorder. J Psychiatr Res 46:1293-1299

Phillips KA, Albertini RS, Rasmussen SA (2002) A randomized placebo-controlled trial of fluoxetine in body dysmorphic disorder. Arch Gen Psychiatry 59:381-388

Phillips KA, Grant J, Siniscalchi J, Albertini RS (2001) Surgical and nonpsychiatric medical treatment of patients with body dysmorphic disorder. Psychosomatics 42:504-510

Phillips KA, Hart AS, Simpson HB, Stein DJ (2014) Delusional versus nondelusional body dysmorphic disorder: recommendations for DSM-5. CNS Spectr 19:10-20

Stangier U, Heidenreich T (2004) Die Liebowitz Soziale Angst-Skala (LSAS). Beltz, Weinheim

Stangier U, Hungerbühler R, Meyer A, Wolter M (2000) Diagnostische Erfassung der körperdysmorphen Störung: Eine Pilotstudie. Nervenarzt 71:876-884

Toh WL, Castle DJ, Mountjoy RL et al (2017) Insight in body dysmorphic disorder (BDD) relative to obsessive-compulsive disorder (OCD) and psychotic disorders: revisiting this issue in light of DSM-5.Compr Psychiatry 77:100-108

Veale D, Gledhill LJ, Christodoulou P, Hodsoll J (2016) Body dysmorphic disorder in different settings: a systematic review and estimated weighted prevalence. Body Image 18:168-186

Vigne $P$, de Menezes GB, Harrison BJ, Fontenelle LF (2014) A study of poor insight in social anxiety disorder. Psychiatry Res 219:556-561

Wittchen HU, Zaudig M, Fydrich T (1997) Strukturiertes klinisches Interview für DSM-IV: SKID; eine deutschsprachige, erweiterte Bearbeitung der amerikanischen Originalversion des SCID. Hogrefe, Göttingen

World Health Organization (2018) International classification of diseases for mortality and morbidity statistics (11th Revision). https://icd. who.int/browse11/l-m/en. Zugegriffen: 8. Jan. 2020

\section{Exzessives Sporttreiben bei Essstörungen}

\section{Psychologische Mechanismen entschlüsselt}

Anhand elektronischer Tagebücher hat ein Forschungsteam des Karlsruher Instituts für Technologie (KIT) und der Universität Freiburg nun erstmals psychologische Alltagsmechanismen aufgedeckt, die dem pathologischen Sporttreiben zugrundeliegen. Die Ergebnisse lassen den Schluss zu, dass essgestörte Personen Sport dazu nutzen, bedrückende Stimmungslagen und negative essstörungsbezogene Gedanken zu regulieren.

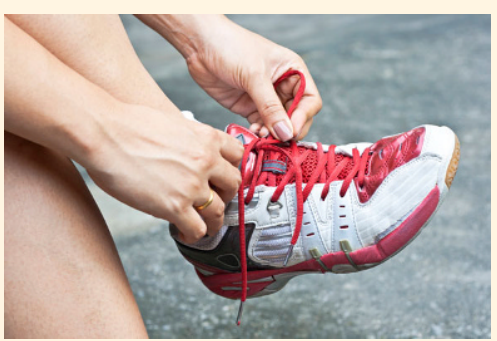

@ e ekapong laksanapiya / iStock / Thinkstock

Die Methode, die diese Studie möglich machte, heißt »Ambulantes Assessment». Sie erfasst menschliches Erleben und Verhalten im Alltag und wurde von einer Expertengruppe am IfSS entwickelt und eingesetzt.

Mithilfe dieser Methodik wurde die körperliche Aktivität von 29 Patientinnen mit Essstörungen und 35 gesunden Kontrollprobandinnen per Accelerometer, einem Bewegungssensor, objektiv und kontinuierlich in deren Alltag über sieben Tage hinweg aufgezeichnet. Der Accelerometer war via Bluetooth mit einem Smartphone verbunden, eine installierte App löste beim Überund Unterschreiten bestimmter Aktivitätsschwellen Tagebuchabfragen aus und die Testpersonen wurden zusätzlich aufgefordert, sowohl vor als auch nach dem Sporttreiben über ihre Befindlichkeit zu berichten. Dabei stellte das Forschungsteam fest, dass bei Patientinnen mit Essstörungen dem Sporttreiben ein Stimmungsabfall vorausging. Dieser Effekt zeigte sich bei den gesunden Kontrollprobandinnen nicht, vielmehr fühlten sie sich vor dem Sport besonders energiegeladen. Nach dem Sporttreiben waren die Patientinnen mit Essstörungen im Vergleich zu den gesunden Kontrollprobandinnen und relativ zu ihrer durchschnittlichen Stimmung besser gelaunt, fühlten sich entspannter, verspürten weniger Druck, schlank sein zu müssen, und waren mit ihrem Körper zufriedener. Dieser Effekt hielt aber nur für eine begrenzte Zeit an, je nach Probandin von circa einer Stunde bis zu drei Stunden. Die Ergebnisse, die das Autorenteam in der Fachzeitschrift Psychotherapy and Psychosomatics veröffentlicht hat, lassen den Schluss zu, dass Patientinnen mit Essstörungen das Sporttreiben dazu nutzen, um bedrückende Stimmungslagen und negative essstörungsbezogene Gedanken zu regulieren. „Um mit schwierigen emotionalen Zuständen und negativem Körpererleben umzugehen, treiben sie Sport, vermutlich auch mangels fehlender alternativer Strategien in solchen Momenten“, erläutert Markus Reichert. „Naheliegend ist auch, dass die positiven Effekte des Sporttreibens das ungesunde Sporttreiben verstärken - sich nach dem Sport befreit zu fühlen, führt zu erneutem Sportreiben, wenn die Wirkung wieder abklingt. Dies kann dann in einen Teufelskreis hineinführen, in welchem immer mehr Sport getrieben werden ,muss' um sich gut zu fühlen", resümieren Markus Reichert und Professorin Almut Zeeck, Koordinatorin der Studie am Universitätsklinikum Freiburg. Laut Zeeck liefern diese Erkenntnisse wichtige Implikationen für die Therapie und weitere Forschung.

Zur Originalpublikation: Markus Reichert, Sabine Schlegel, Friederike Jagau, Irina Timm, Lena Wieland, Ulrich Ebner-Priemer, Armin Hartmann and Almut Zeeck: Mood and Dysfunctional Cognitions Constitute Within-Subject Antecedents and Consequences of Exercise in Eating Disorders. Physotherapy and Psychosomatics. DOI: 10.1159/000504061.

\section{Quelle: KIT - Die Forschungsuniversität in der Helmholtz-Gemeinschaft, 30.01.2020 (www.kit.edu/kit)}

